\title{
Water research paradigm shifts in South Africa
}

AUTHORS:

Raymond Siebrits

Kevin Winter ${ }^{1}$

Inga Jacobs ${ }^{2}$

\section{AFFILIATIONS:}

${ }^{1}$ Environmental and Geographical Science, University of Cape Town, Cape Town, South Africa ${ }^{2}$ Water Research Commission, Pretoria, South Africa

\section{CORRESPONDENCE TO:} Kevin Winter

EMAIL:

kevin.winter@uct.ac.za

\section{POSTAL ADDRESS:}

Environmental and Geographical Science, University of Cape Town, Private Bag X3, Rondebosch 7701, South Africa

DATES:

Received: 18 Sep. 2013

Accepted: 25 Nov. 2013

\section{KEYWORDS:}

research; paradigms; South Africa; horizon scanning; scientometrics

\section{HOW TO CITE:}

Siebrits R, Winter K, Jacobs

I. Water research paradigm shifts in South Africa. S Afr J Sci. 2014;110(5/6), Art. \#2013-0296, 9 pages. http://dx.doi.org/10.1590/ sajs.2014/20130296
We performed a scientometric analysis of water research publications extracted from four decades of South African related papers to identify paradigms and paradigm shifts within water research in South Africa. Between 1977 and 1991, research publications are dominated by research into technical and engineering solutions, as well as designs and plans to secure water supply. From 1992 to 2001, publications on water pollution, water quality, water resource management and planning are prominent. The second major paradigm is observed from 2001 to 2011 in which the emphasis is on planning, modelling, catchment-scale studies and a multidisciplinary approach to research. Another transition period, towards the end of 2011, is characterised by uncertainty, although it also shows the prominence of key concepts such as participation, governance and politics in water management. The second aim of this study was to identify and prioritise current and future water research questions through the participation of a wide range of researchers from across the country, and to relate these questions to research paradigms, issues and concerns in water in South Africa. Over 1600 questions were collected, reduced in number and then prioritised by specialists in the water sector. The majority (78\%) of questions offered by respondents in the South African case study dealt with relatively short- to medium-term research requirements with $47 \%$ of questions focused on medium-term issues such as supplying water, service delivery and technical solutions.

\section{Introduction}

Limited historical data are available to describe water research in South Africa over the first half of the 20th century. Many authors recognise that this period was dominated by technological developments, breakthrough research and projects in water storage and transfer, as well as the positivist approach to nature and development..$^{1-3}$ For example, the development of irrigation in agriculture played a major role in shaping early 20th century water policies, infrastructure and socio-economic development in South Africa. ${ }^{2}$

A new era in water research in South Africa began with the promulgation of the Water Research Act No. 34 of 1971. The Act led to the formation of the Water Research Commission (WRC) and the Water Research Fund with the purpose of initiating, managing and financing water research. The objectives of the WRC, as stated in the Act, were to co-ordinate, promote and encourage research in respect of a wide range of purposes and activities. ${ }^{4}$

Furthermore, the National Water Act No. 36 of 1998 contributed to both forging radical changes in water resource management and new directions in research. ${ }^{5}$ This legislation replaced many previous inconsistent acts that focused on water security, supply-side interventions and water as an economically exploitable resource. A shift in the political landscape, marked by the first democratic elections in South Africa in 1994, contributed to a major shift in the existing water resource management paradigm. Legislative reform was timeous amidst growing concerns about the state of the country's waterways and the rising capital expenses in supply schemes, coupled with growing environmental concerns globally. ${ }^{6-8}$ South Africa is lauded as being the first country in the world to have promulgated national water legislation which uses water to achieve societal transformation and focuses attention on environmental and social justice. ${ }^{7}$ However, although the National Water Act introduces a paradigm shift towards a socially equitable and just resource management society, ${ }^{9}$ South Africa continues to experience a water deficit that requires urgent management, mitigation and interventions.

We have identified the prevailing paradigms that have influenced the history of water research in South Africa by analysing the publication output over the last four decades. In identifying research questions proposed by a range of researchers active in the water sector in South Africa, we also aim to gain insight into future water research questions and approaches.

One of the challenges lies in finding acceptable methods of identifying paradigms and interpreting these through an historical analysis as well as in contemporary times. The bulk of the theoretical and methodological arguments for question prioritisation, as well as further detailed results and discussion, can be found in Siebrits et al.${ }^{10} \mathrm{~A}$ brief overview of the processes is provided along with the final results: the priority water research questions for South Africa formulated in the study completed in 2012.

\section{Emerging paradigms in water resource management}

A paradigm can identify a conceptual framework that is composed of a class of common elements, theories, laws and generalisations that is widely acknowledged within a scientific school of thought or discipline. Paradigms also shift for a variety of reasons and under various influences. According to Kuhn ${ }^{11}$, when enough significant anomalies have accrued against a current paradigm, then the scientific discipline is thrown into a state of crisis. During this crisis, new ideas, and even those previously discarded, are tested further. A paradigm shift occurs only after a given discipline or significant thinking in a field of knowledge changes, and only when this change is widely recognised. Sometimes paradigms only gain ground because of some dramatic and unforeseen verification, such as a shift in legislation or policy, or for personal or aesthetic reasons in which they may appear neater, simpler or more elegant than their older counterparts do.

When new paradigms appear, however, they are rarely complete. More often, they are the products of relatively sudden and unstructured events that arise from an enlightened moment in which previously hidden components of knowledge suddenly come into view. ${ }^{11}$ New knowledge sometimes has the power to cause an anomaly leading to 
an unexpected change in the world view of those holding one or another paradigm. Thus, a change of world view begins when a significant anomaly is recognised within an existing paradigm. The challenge is often to identify the anomaly and then to recognise the significance or importance of the phenomenon. The signals and changes in paradigms, with respect to paradigm changes in water resource management, provide the context in which to explore corresponding changes in the water research enterprise in South Africa.

One of the earliest paradigms in water resource management began at the start of the 20th century and is most often acclaimed as the hydraulic mission because it is characterised by major engineering activities involving the construction of water infrastructure to capture, store and distribute water. This period is also described as the heroic engineering phase ${ }^{12}$ which is noted for its immense scale of projects and plans. This phase also finds support in modernist or positivist philosophical beliefs which consider it possible to control and manage nature. The majority of water projects in this period were concerned with supplying more water, more efficiently, to more areas. ${ }^{1,13}$

The demand side of water resource management, which is also a management paradigm, focuses attention on how to manage water demand and use. This shift is influenced to an extent by various social advocacy movements, but is also driven by increasing recognition of resource scarcity, heightened interest in sustainable development considerations, post-modern philosophies and increased prominence of environmental justice, equity and democratisation of resources. ${ }^{1,14}$

On a larger, global scale, Allan ${ }^{15}$ makes further use of paradigms in explanations of global changes in water resource management. His work focuses on the development of an analytical method to address the problem of water resource allocation. Allan's contribution lies in identifying paradigms that are reliant on economic, legal and political factors that influence the water sector in semi-arid countries. This shift in paradigms is represented in a transition of five water management paradigms, each with its own distinct focus and function.

The first of the five paradigms is referred to as the pre-modern paradigm, which spanned from 1850 to the beginning of the 19th century, and which was dominated by a general increase in water supply and usage. It occurred in an era of the hydraulic mission in which ingenuity and engineering efforts abounded. ${ }^{12}$ The second paradigm from the early to late 20th century was characterised by industrial modernity and again featured an increase in activity in the hydraulic mission. In this phase, water demand increased because agricultural activity shifted from subsistence to commercial-based economies, followed by further demands on water resources as a result of the rapid increase in industrial activity.

The third paradigm in Allan's $\mathrm{s}^{15}$ framework, which occurred in industrialised nations from the 1960s, shows a shift towards sustainable resource management and a concerted effort to redress the damage done by previous paradigms. The fourth is characterised by a period of economic expansion (particularly in the North) and by smart economic decisions that offer several environmental advantages, but is also characterised by a general decline in the hydraulic mission. Finally, the fifth paradigm is dominated by political and institutional change which becomes increasingly aligned with global shifts towards sustainability and also a rapid decline in the hydraulic mission.

Overall, there are elements within Allan's management paradigms that are in alignment with similar developments in water resources in South Africa. The assumption too is that the water research enterprise in South Africa corresponds reasonably well with these changing paradigms in water resource management. The occurrence of different paradigms, as suggested by Allan, especially the third, fourth and fifth, are of particular interest in this study because these paradigms represent a period in which the research effort should be detectable in the research publication evidence and in the formulation of research questions that was undertaken as a part of this study.

\section{Paradigms in research publications: A scientometric analysis}

Given the evolution of water management paradigms in South Africa and also globally, the question then arises as to whether scientific research has responded to these paradigmatic changes, and if so, in what ways. Has research driven these management paradigms or has it lagged behind?

The field of bibliometrics provides useful insights into the health of a country's national innovation system and is also a necessary and integral part of science policy. For the purposes of this paper, bibliometrics is synonymous with scientometrics, that is, the analysis of science and scientific output. Scientometrics is used interchangeably with bibliometrics and informetrics. ${ }^{16}$ The three fields all refer to the study of science, knowledge, and knowledge management and production. In this study, scientometrics is considered as the 'study of the quantitative aspects of science as a discipline or economic activity'17. It is used to analyse the evolution and academic output of water research within a socio-political and historical context.

Scientometric methods begin with the collection of a series of appropriate publications or reference material followed by a network analysis. Sets of keywords and/or noun phrases amongst the journal articles are analysed with respect to their frequency to each other within an article and between articles. This approach results in a topic/word/ concept co-occurrence network. In this analysis, statistical algorithms, such as cluster analysis and multidimensional scaling, are used as the foundation of scientometrics.

Studies have tested the strength of accepted scientometric methods and showed that scientometrics is robust and reliable even on a coarse level. ${ }^{18,19}$ Arguably, the main societal impact of scientometrics has been the creation of the impact factor and analysis of research, researchers, publications and journals.

Scientometric maps have a number of advantages in the output of research analysis. Researchers argue that scientometric maps are an important means of conveying the results of the method. ${ }^{20,21}$ The maps allow for the representation of diverse and large sets of data, but they remain heuristic tools that can be used to explore and consider plural perspectives to inform decisions and evaluations. ${ }^{21}$ In this study, maps are the main means of representing results of patterns and trends in water research in South Africa.

\section{Identifying water research questions}

Scientometric analysis of published works provides an interpretative account that helps to identify patterns of change and to understand the relationships that influence these trends. However, scientometrics is not an appropriate method for determining future water research questions. For this purpose, we used a form of horizon scanning to identify future research questions and strategies using methodological elements similar to studies undertaken by Sutherland and Woodroof ${ }^{22}$ which are to: (1) scope the issue, (2) gather information, (3) spot signals, (4) watch trends, (5) make sense of the future and (6) agree on the response. In this study, we used a similar approach which is supported by a collaborative, multi-stakeholder process to identify and examine threats or trends in society, the environment or a sector, and to identify needs that will enable appropriate questions..$^{22,23}$

\section{Research methods}

\section{Scientometric analysis}

A conceptual narrative on water research in South Africa is central for the discussion on water research paradigms, knowledge and appropriate adaptive capacity. Many authors have discussed how these approaches provide an objective and evidence-based means of assessing the state of a research or scientific field $16,24,25$. Scientometric methods are based on two assumptions. Firstly, that 'scientific knowledge can be represented as a network of concepts or ideas, and that these elementary entities can be aggregated to form macro-structures' ${ }^{\prime 26}$. Secondly, if mapped or represented in a structured manner then 'it is assumed that each map is a snapshot at a distinct point in time of what is actually a changing and evolving structure of knowledge'26. The key data for this method are research outputs, in the form of publications, collaborations, intellectual property, policy influence and applications. 
Locating relevant water-related publications objectively and comprehensively is a challenge within itself. This challenge stems from the definition of water research used herewith. In this study, the journal search set comprised a twofold approach. Firstly, journals that published five or more articles with the search terms 'water' and 'South Africa' (or derivatives thereof) were included. Secondly, snap polls and pilot surveys undertaken towards the end of 2011 that included questions asking practitioners where they published and read South African water-related research were included. The results from the significant publication count criteria and stakeholder input amounted to 171 publications forming part of the journal search set. These journal titles were then added to the query and searched further. The final search query was for journal articles that contained 'water' and 'South Africa' in their topic within the journal search set. Searches were performed on Thomson Reuters Web of Science.

Scientometric queries require that the content or topic of research outputs must be analysed. The most widely used method for this analysis is the co-word analysis of research publications, particularly within their title and abstract. ${ }^{25,27-30}$ The methodological foundation of coword analysis is the idea that the co-occurrence of words describes the contents of documents. By measuring the relative intensity of these co-occurrences, it is possible to establish a simplified representation of a field's concept networks. ${ }^{27} \mathrm{C} 0$-word analysis examines the frequency of individual words or word phrases within a data point (publication) and concurrently across the data set. The more frequently a word or phrase appears in a data point, the more relevant that topic becomes within the data points.

The final output from this process is a network visualisation file (.net) for the most frequent keywords within the data set and their relative frequency towards other keywords. This network file is the fundamental output of mapping and visualising networks within scientometrics. The most common method of visualising or representing these outputs is through network maps. These maps are simplistic representations of the networks themselves and represent the strength of topics and their interrelationship (associated strength) with other topics. The majority of network maps use size and distance as indicators of certain attribute properties or relationships.

There are limitations in the use and interpretation of scientometric maps because the output provides only a representation of relationships among terms found in published content. The results should be interpreted with caution even though the evolution of scientometric methods represents the most effective known method of simply representing scientific relationships, output or developments on a particular scale.

\section{The search for water research questions}

A form of horizon scanning was used in this study to identify and evaluate research questions that are currently being asked by researchers. There are three main methodological steps that are typically used: (1) identify and create a collaborative stakeholder network, (2) collect data from this network regarding research expertise, opinions on research considerations and research questions and (3) analyse this data by allowing the network to deliberate the results and produce a final set of results of research opinion and questions.

A substantial taxonomy of horizon scanning methods used in identifying and prioritising future research questions, scenarios and needs is provided by Sutherland and Woodroof ${ }^{23}$. They follow a combination of open fora, trend analysis, questionnaire and expert consultation. There are multiple reasons for attempting to engage a wide variety of stakeholders. Arguably a strength, and at the same time a weakness of the current study, was the intention to involve a wide variety of stakeholders with an interest in water and water research, and to engage these participants through the voice of a research initiative, rather than through that of the researchers. The intention was to make communication professional, allow for branding to be created, and to enable a common identity if other researchers began working on, or in association with, the project. The detailed methodological steps and substantial outputs from this process can be found in Siebrits et al. ${ }^{10}$ while relevant results are presented here.

\section{Results and analysis}

\section{Research output}

The number of journal articles and research reports published per year from 1977 to 2011 as identified through the search is shown in Figure 1. The stacked column graphic shows the proportion of WRC research reports, Water $S A$ articles and other journal articles. In summary, there is an increase in annual publication counts, a rise in Water $S A$ articles and a marked increase in WRC research reports. The increase in the proportion of other journal articles from the early 1990s until the present is notable. South Africa's water-related research output has steadily increased and the research is found in more diverse, international journals.

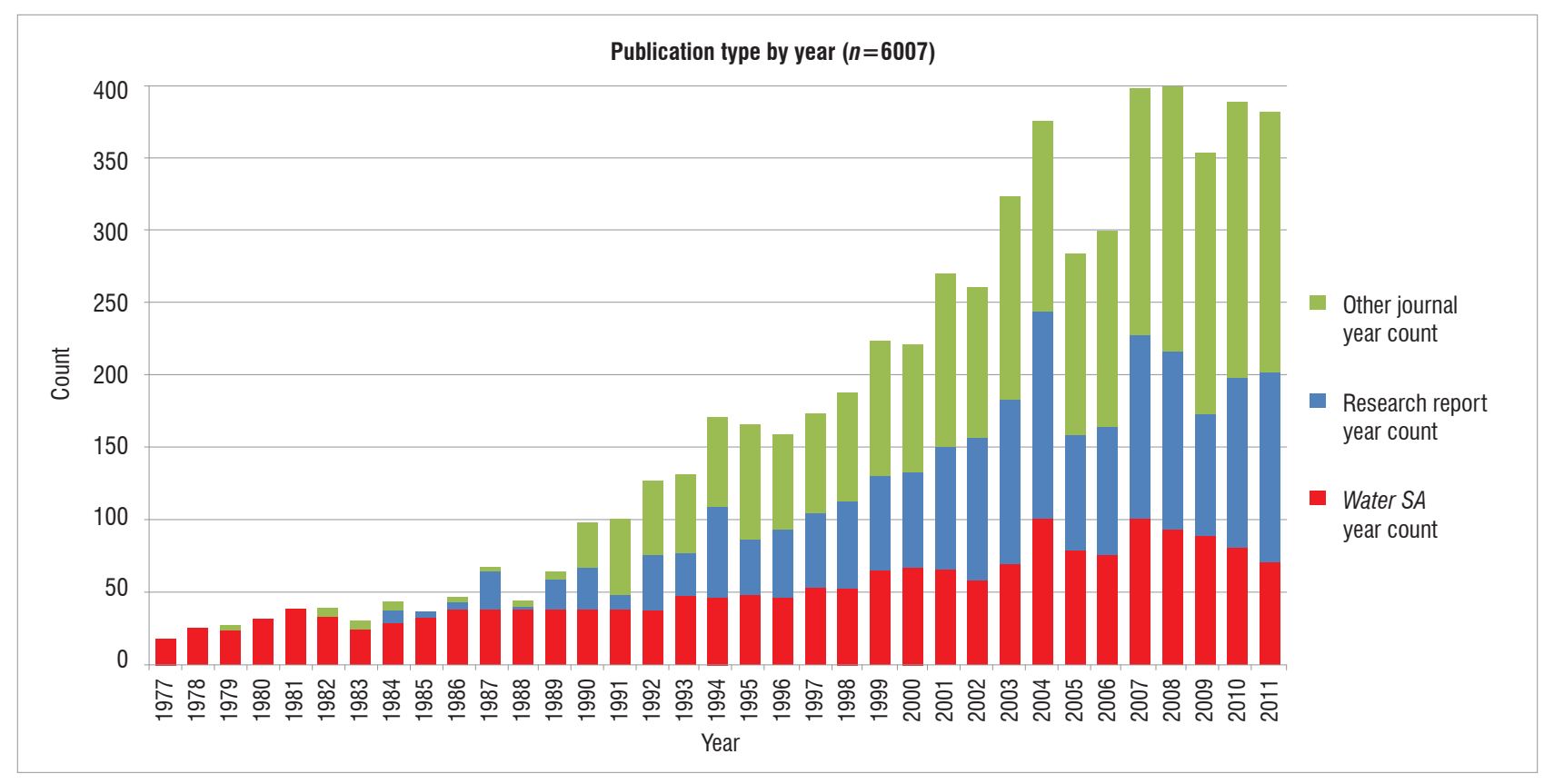

Figure 1: Publication type by year for all data points and all data sources. 
A scientometric map was created using Sci2 and VOS viewer and is displayed for illustration purposes in Figure 2. The figure comprises results for a specific time slice, shown in label and density format. Label format presents more prominent words in the network as larger spheres. The closer spheres or words are to each other the more interrelated they are in the network. Colours represent clusters or sets of related words as they emerged from the network (i.e. more general relationships). Lines represent significant connections between words of the same or different clusters. Density format presents the identical map but uses warmer colours to display words and clusters of greater prominence with the colour contours conforming to how strongly related clusters are. Word size represents general prominence as per label view.

The map shows the time slice from 2002 to 2006 (Figure 2). It illustrates a range of emerging research fields and a general increase in overall publications. The word 'management' becomes pronounced along with terms such as 'community', 'impact' and 'application'. It is also during this phase that the word 'integrate' becomes increasingly prominent.

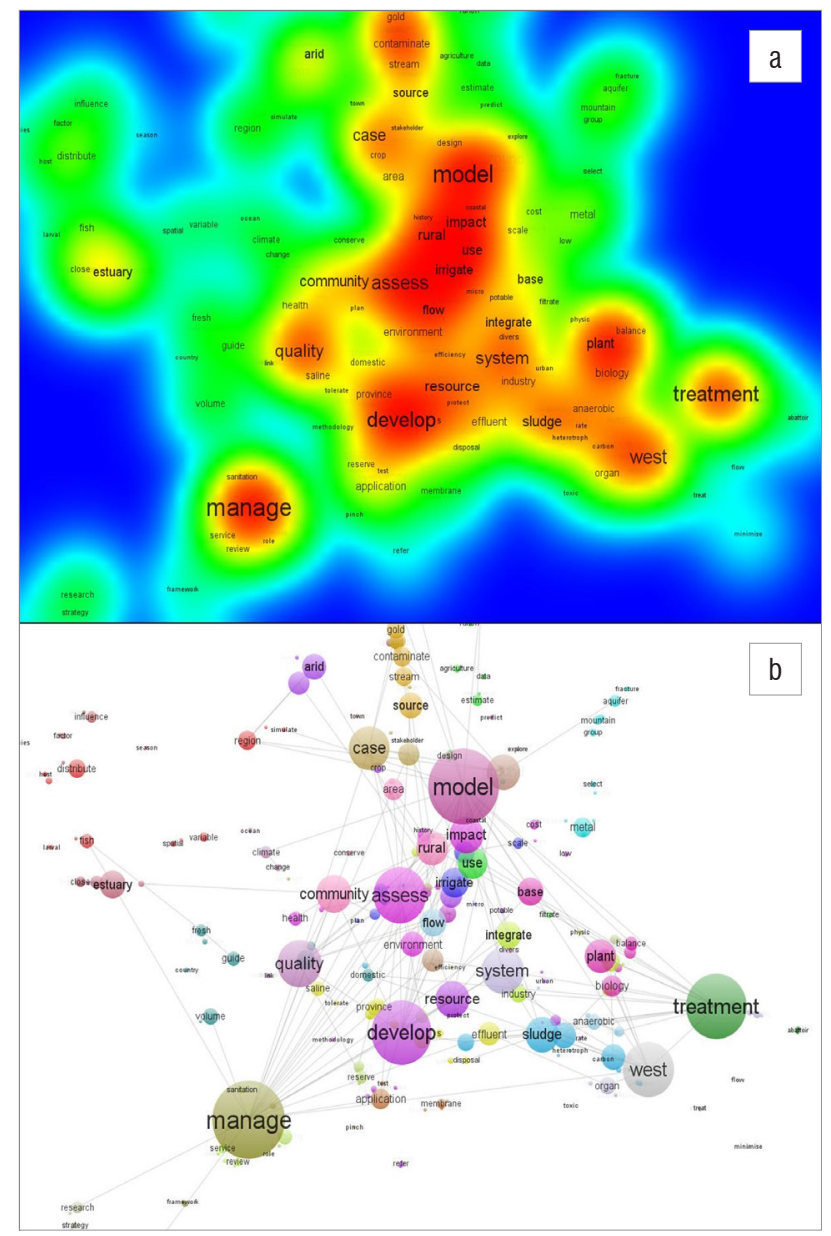

Figure 2: (a) Density and (b) label format bibliometric maps based on keywords in publications from 2002 to $2006(n=1545)$.

The stakeholders captured by the research signed up and engaged in the process for numerous reasons. Some simply wanted to remain informed of the process and results. Some saw an opportunity to participate in the surveys and discussions while others used the portal to seek further information about water research. When the study was completed in December 2012, there were 2260 unique stakeholder contacts on the database.

The stakeholders contained within the database were diverse in their involvement in the South African water sector but appeared well connected within the water sector networks. Figure 3 displays the organisations represented in the database. Overall, stakeholders in the database were affiliated to 572 organisations or institutions.

\section{Question gathering}

Overall, 1075 stakeholders were contacted directly via individual telephone calls during May and June 2012 to be alerted about the survey. By the time the main survey closed in December 2012, 641 completed responses had been received. Of the 1674 questions submitted, 4629 keywords/categories were provided of which 844 of these were unique. These keywords/categories can be seen as the descriptive data of the submitted questions and guided the identification of themes for the workshop. Table 1 shows the top 40 keywords in the data set along with the number of counts per keyword in brackets.

The most striking result is the 245 occurrences of the keyword 'management'. A large proportion of the submitted questions had a management-oriented line of inquiry. The questions were further categorised into six themes:

1. Change - building socially resilient and adaptive responses to social, climate and general environmental change

2. Data - capturing of quality data through strategic monitoring, and with reliable analysis, modelling and scientific reporting

3. Ecosystems - protection, conservation, restoration and productive use of healthy ecosystem services

4. Governance - integrated, strategic adaptive management

5. Innovation - investment in infrastructure and research for innovation

6. Resources - protection, conservation, treatment and management of water resources for equitable growth and development

Following further refinements to the questions, which included filtering for duplicates and suitability as well as quality control through reasonable testing, a total of 401 questions were presented as the input data to the Water Research Horizon Scanning Workshop in October 2012 in Cape Town. Delegates were tasked to reduce the 401 questions to approximately an eighth in total number. The final data set amounted to 59 priority water research questions across the six themes. The workshop question prioritisation which constituted the central output result from the workshop is presented in the publication by Siebrits et al. ${ }^{10}$

\section{Research output and links to paradigms}

South Africa has undergone significant changes in the output and structure of water research over the past four decades. There has been substantial growth in output with a total relevant sample publication record of 6007 articles and research reports and a current annual output of over 350 articles and reports per year. The number and sources of journal articles over this period have increased and diversified while WRC research report output has also increased, albeit at a slower rate.

The emergence of two main areas of research or fields of specialisation in the democratic transition (1992-1996) period is supported by greater diversity of publications than in previous years. The engineering or technical research outputs cluster together and again focus on treatment systems, processes and evaluation. This time the clustering is associated with management-based and planning-oriented research which is pronounced in the words 'catchment', 'develop' and 'urban'. Although somewhat dispersed, water quality and algae also emerge as topics of research concern.

A transition period in water research occurred over a period that became increasingly focused on quality constraints, fields of management and planning. Words such as 'review', 'model', 'community' and 'geography' begin to appear in the research publications. The emergence of these words supports the beginning of paradigm changes as a result of water deficits towards end-use efficiency as outlined by Ohlsson and Turton ${ }^{14}$. The beginning of paradigm changes also indicates that the second transition of Turton and Meissner ${ }^{31}$ occurred with a new social contract around water that came not only from a new political regime and democratic transition that focused on redistribution, but also one that was spurred on by a movement of South African environmentalism, the beginning of the global sustainability debate and the rise of civil 


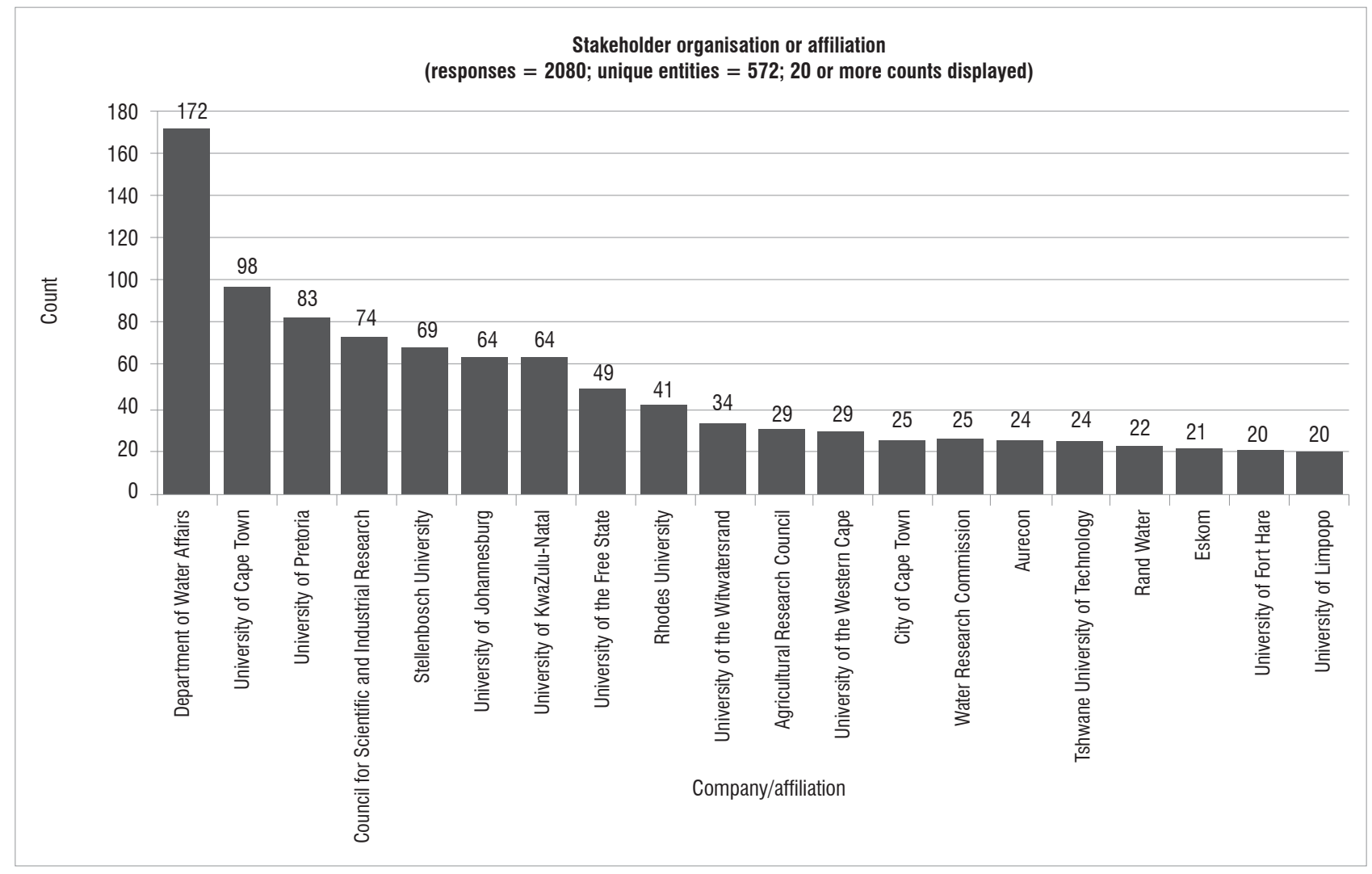

Figure 3: Distribution of stakeholder organisation or affiliation.

Table 1: Top 40 research questions by keyword provided by stakeholders

\begin{tabular}{c|c|c|c|c}
\hline management (245) & groundwater (79) & sanitation (54) & technology (44) & conservation (27) \\
\hline treatment (136) & hydrology (73) & services (53) & policy (43) & capacity (26) \\
\hline quality (118) & mining (73) & education (51) & usargy (26) \\
\hline supply (103) & health (72) & research (50) & wetlands (34) & planning (26) \\
\hline wastewater (99) & economics (65) & monitoring (49) & environmental (30) & urban (26) \\
\hline agriculture (94) & catchment (55) & resources (49) & industry (30) & waste (25) \\
\hline pollution (83) & change (54) & ecology (47) & demand (29) & alternatives (25) \\
\hline governance (80) & climate (54) & river (45) & & \\
\hline
\end{tabular}

The number of counts per keyword is indicated in brackets.

society activism. Here marginal uncertainty begins to creep into the understanding of water affairs as described by Allan ${ }^{15}$ and the need to model, plan around catchments and include other disciplines (especially from the humanities) begin to become considerations in the research environment.

The period 1997-2001, around the major transformation of South Africa's water laws and post-establishment of the national Constitution, shows a strong polarisation between the main technical and managementorientated disciplines. Words such as 'develop', 'manage' and 'assess' become more prominent while the technical focus diminishes. Researchers began to focus further on understanding the broader water context, to use systems approaches and to begin to plan for more than just engineering solutions. These results support the view that a transition was still underway with regard to the dominant paradigms, but the word system had shifted noticeably towards the management- and development-related research disciplines and away from the technical.

The most recent decade of water research represents the greatest change in water research paradigms. It represents over half (3456 of 6007) of the collected and analysed publications, and constitutes the most representative sample of current recent water research. In this period, words become clustered and centralised, with images being most clustered in their centres with few stand-alone concentration areas. This pattern indicates how research has become more diverse yet interconnected and a shift towards other disciplines. This is most prominent in the first series of the millennium analysed (2002-2006) with an emphasis on concepts such as management, modelling and development. These observations point to research that is directed towards dealing with current issues and societal benefits and needs. 
Between 2007 and 2011 there appears to be a significant interconnectedness of specific keywords with many others. Here management has become a key research theme, which is connected to almost every other keyword or area of interest. All major areas of water research received fair attention and prominence in the results, from treatment systems to catchments, modelling, communities, development and biological concerns. The word 'integrated' is increasingly prominent and linked to management, suggesting a dominant thrust in water research activity over this time. The growing prominence of climate-related research also highlights growing global interest in environmental change. Another interesting emerging field is groundwater research. While this topic has been present alongside general hydrological keywords and concepts, during this period the development and impact of the groundwater theme appears to become more independent than before.

The research effort in South Africa appears to have evolved into a new set of paradigms, albeit it tentative and uncertain, in which some emphasis is given to the social sciences disciplines and to concepts of governance and management. There is also evidence of research that focuses more attention on demand-side applications and interests, and integrated management. However, a third or reflexive transition phase does not appear just yet. ${ }^{15}$ Keywords that relate to the green economy or risk awareness are not yet prominent. What is obvious is an increase in the prominence of collaboration across multiple disciplines over the last decade.

In brief, the scientometric analysis of South African published works on water research over the past four decades shows two reasonably distinct paradigms (Figure 4). The first paradigm occurs in a period dominated by the quest to supply water, which is interrupted dramatically by changes in the political landscape. The Constitution, the National Water Act among others, and the shift in the national balance of power, introduce the next paradigm shift and an emphasis on integrated water resource management. This new paradigm is characterised by a research effort that is centred on new themes and concepts such as sustainability, community, governance and adaptation. The shift from the 1980s, once dominated by research efforts that focused on treatment, technical interventions, chemistry and so forth, now features research interests, themes and approaches such as integrated water resource management and multidisciplinary studies in water research.

Finally, it is interesting to observe what is not prominent in the scientometric results. Topics and themes such as data quality and integrity, law, rights, access, licensing and culture are noticeably absent from most of the scientometric outputs. This absence does not necessarily mean that they are being ignored, but rather that they are receiving less attention than other research disciplines and specialisations. The absence of these terms does not necessarily alter the observed paradigmatic shift, but may suggest that the South African water research field is not yet ready to move on to another water paradigm - at least not in the immediate future.

\section{Identifying and prioritising questions and the link to paradigms}

The launch and strategies undertaken through the Aqua d'UCT initiative far surpassed expectations with regard to participation, uptake and response. The robust and yet diverse nature of the results and community interaction during the study was shown by the steady growth of interest from approximately 600 to over 2000 stakeholders on the research contact database by the time the study was completed in 2012 .

The most salient findings of the survey indicated that many respondents wanted longer and more substantial research projects to be funded and established, yet the majority of research questions that were offered were categorised as short- to medium-term projects taking only 1 to 3 years to complete. Nevertheless, these questions reflect the diverse research disciplines and specialisations as suggested by the keywords such as 'management', 'governance', 'planning', 'education', 'policy', 'planning' and 'alternatives' being most prominent. However, those questions of a more technical nature relating to treatment, quality and pollution, hydrology, climate, supply and ecology dominate the input data set.

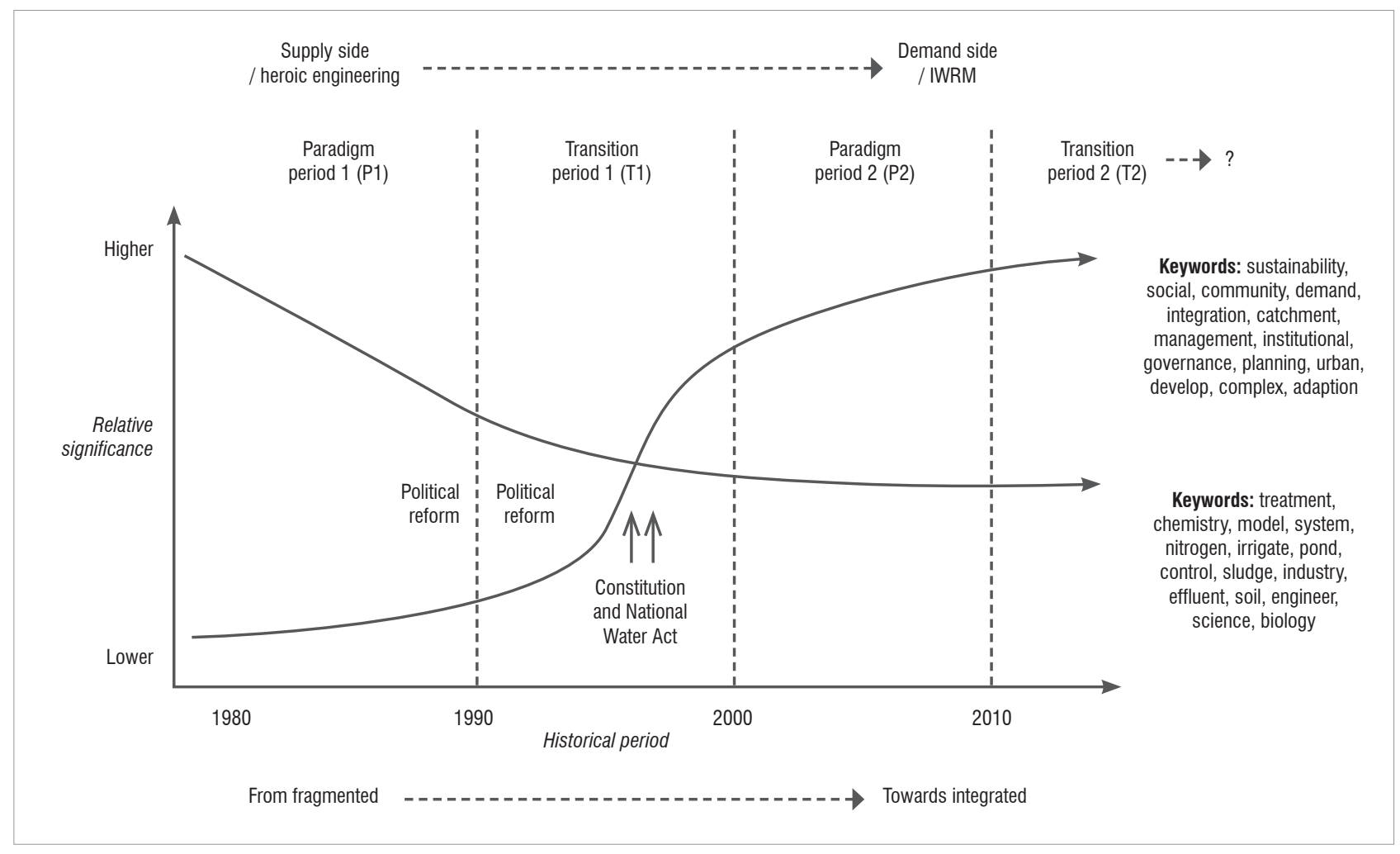

IWRM, integrated water research management

Figure 4: Paradigms and transitions emerging from scientometric analyses. 
The survey results form a substantial collection of research questions from water research stakeholders. The process of reducing the survey data set into something manageable for prioritisation at the final workshop was also a rigorous one. The reduction from 1603 initial to 59 priority research questions followed similar methods to those used by Sutherland et al. ${ }^{32}$ The only significant change was to gather the initial data set of questions from a broad and larger community rather than from key specialists only.

As mentioned earlier, the final set of research questions is presented in Siebrits et al. ${ }^{10}$ Here we have analysed the content of the questions further and organised the results into two categories. The first category is a combination of words and concepts from each question placed in three columns - short-, medium- and long-term research challenges. The method is a subjective one. Some researchers will interpret the questions differently according to factors such as context and experience. In addition, no question resides exclusively within a particular level of challenge. For example, a short-term research question that seeks to understand the skills gap in the water sector might identify the extent of the problem and the causal factors, but skills development is more likely to be a long-term issue that requires careful monitoring, evaluation and interventions that are informed by research that falls into a long-term research challenge.

The second category organises the questions into indicators of knowledge and knowledge management. For example, the solutions to many research questions are already known from previous studies conducted elsewhere in South Africa or in the world, whereas questions dealing with long-term issues and grand challenges require a much greater commitment to knowledge construction.

Table 1 maps all 59 questions against this matrix. The results were not tested with the stakeholders, but are presented as a contribution to thinking about how best to incorporate multiple criteria into the development and organisation of the research question bank.

In general, the presentation (Table 2) confirms three important observations. Firstly, over $78 \%$ of the questions that were offered and refined at the workshop seek to address short- to medium-term research questions, typically questions dealing with service delivery, sanitation, access to water, pricing and water quality. Secondly, the majority of the questions confirms the existence of a transition paradigm, similar to what was identified earlier in the scientometric analysis. These questions deal with issues of intermediate concern but are also dominated by issues of integration, data and information systems, social change, planning decisions and further development of regulations. The majority of the questions is organised in the medium-term category. The uncertainty is created partly by the long list of questions that seek to address multiple issues that beset the South African water research landscape. Finally, there is a small set of questions that is arguably more closely aligned with issues and concerns that features some elements of Allan's $\mathrm{s}^{15}$ third, fourth and fifth paradigm. Here the questions deal with medium- to longterm critical concerns of sustainability, establishing green economies and implementing new forms of integrated, adaptive governance. These kinds of questions pose extraordinary challenges necessitating considerable financial and institutional support. Two examples of these kinds of questions are:

- How can innovative process technologies, including nanotechnology, be applied to benefit water and wastewater treatment process?

- What are the life cycle and systematic impacts of acid mine water and how can these be managed, mitigated, remediated and beneficiated?

Delegates acknowledged that the workshop was an energising and interesting collaborative exercise. While there were some obvious

Table 2: Categorisation and organisation of the research question data set

\begin{tabular}{|c|c|c|c|}
\hline $\begin{array}{l}\text { Research questions: } \\
\text { Extent of challenge }\end{array}$ & $\begin{array}{l}\text { Short term } \\
\text { (immediate: } 2 \text { to } 3 \text { years to complete } \\
\text { these projects) }\end{array}$ & $\begin{array}{l}\text { Medium term } \\
\text { (10 to } 20 \text { years to complete these projects) }\end{array}$ & $\begin{array}{l}\text { Long term } \\
\text { (grand challenges: more than } 20 \\
\text { years to complete) }\end{array}$ \\
\hline $\begin{array}{l}\text { High level of knowledge } \\
\text { required together with } \\
\text { financial investment, } \\
\text { expertise, capacity, } \\
\text { leadership, etc. }\end{array}$ & $\begin{array}{l}\text { Integrated planning; challenge of rapid urbani- } \\
\text { sation; threat of mining on water quality; poor } \\
\text { regulation; freshwater pollution; access to } \\
\text { water; managing fit of purpose water; rural } \\
\text { water management; impacts of eutrophication }\end{array}$ & $\begin{array}{l}\text { Early warning systems; impacts on health; economic } \\
\text { and social value of ecosystems and services; ecological } \\
\text { reserve determination; sustainable abstraction; } \\
\text { sustainable catchments; applying business model } \\
\text { to catchments; improving treatment of emerging } \\
\text { contaminants; understanding water and energy } \\
\text { nexus; water use in agro-industry; improving footprint } \\
\text { assessment; risks and impacts of acid mine drainage; } \\
\text { building water-sensitive settlements }\end{array}$ & $\begin{array}{l}\text { Food and water security; ensuring } \\
\text { functional, safe, socially just service } \\
\text { delivery; understanding and addressing } \\
\text { global scale change impacts on water } \\
\text { resources; addressing ecological risks } \\
\text { that also impact on society; analysing } \\
\text { emerging micro-pollutants and } \\
\text { treatments; treating pathogens; using } \\
\text { nanotechnology in water treatment }\end{array}$ \\
\hline $\begin{array}{l}\text { Reasonably high level of } \\
\text { knowledge required to meet } \\
\text { these challenges }\end{array}$ & $\begin{array}{l}\text { Challenge of meeting supply and demand; } \\
\text { waterborne sanitation challenges; access } \\
\text { to efficient water-based services; societal } \\
\text { contribution to total water services; open- } \\
\text { access data sources; impact of invasive } \\
\text { species on water resources; skills gaps; water } \\
\text { losses; socially just water pricing; efficient } \\
\text { water use; regulating illegal water use }\end{array}$ & $\begin{array}{l}\text { Enabling relevance of water research; determining } \\
\text { societal and environmental value of water; improving } \\
\text { real-time data capture; using data for decision support } \\
\text { systems; accountability in governance of water; efficacy } \\
\text { in bio-remediation projects; protection of ecological } \\
\text { systems; improving public education and response; } \\
\text { developing innovation in information systems; ring- } \\
\text { fencing water costs; enforcement of water quality; } \\
\text { establishing co-operative governance; improving } \\
\text { communication networks and tools; managing sediment } \\
\text { accumulation; use of remote sensing in monitoring; } \\
\text { addressing agro-hydrological drainage; climate } \\
\text { change and water scarcity and threats; water resource } \\
\text { management in informal settlements; importance of } \\
\text { water in urban design and planning; innovation and } \\
\text { development in water re-use; impacts of shale gas } \\
\text { fracturing; responding to growth and development; } \\
\text { ecosystem protection; policy and legislation response to } \\
\text { scarcity and poor water quality }\end{array}$ & $\begin{array}{l}\text { Analysis, assessment, monitoring, } \\
\text { reporting and regulation of groundwater } \\
\text { at national scale; determining risks } \\
\text { and impacts of using different forms of } \\
\text { treated water for irrigation }\end{array}$ \\
\hline $\begin{array}{l}\text { Relatively easy to build the } \\
\text { required level of knowledge } \\
\text { (much is already known) }\end{array}$ & & $\begin{array}{l}\text { Improving quality and access to hydrological data; } \\
\text { understanding change drivers in water resources } \\
\text { management; transitioning to sustainable development }\end{array}$ & \\
\hline
\end{tabular}


gaps in the representation of participants, delegates were pleased to interact with diverse leaders in the field. Most delegates appreciated the quality of exchange and interaction during the formal and informal activities. Positive comments were also received about the organisation, facilitation, the venue and structure of the workshop. Many said that the structured approach to the workshop made the best use of time in order to achieve the intended product.

The strongest criticism from delegates was that the approach and methods used at the workshop were not designed to identify horizon scanning research questions per se. Rather, delegates said that they felt coerced into responding to questions that were put before them. Moreover, delegates felt that it was difficult to develop new questions that were of an horizon scanning, long-term nature for a number of reasons: the groups were too diverse, there was insufficient time to consider and develop meaningful questions and the process was too demanding for the facilitators which resulted in tasks being carried out in a mechanistic manner against a tight time frame.

Delegates were also critical of the fact that they had to work with a large number of questions that were poorly formulated. Problematic questions came in a number of forms: they were often about immediate issues; they were not valid research questions; they were too broad to be categorised in a chosen theme; they were often limited to disciplines and fields within the natural sciences; and many did not show any insight into what might lie on the horizon.

The final list of questions may well be indicative of the current state of thinking amongst researchers generally. The majority of the questions focused on end-use efficiency, demand-side management and technical solutions, with only a few dealing with research to further innovation, progressive forms of governance, and the integration of other sectors and their respective alignment with water resource management. This apparent lack of creativity and innovative thinking also explains the frustration that some delegates felt in that they were expecting to be able to provide foresight into drafting new types of questions that would create a new set of paradigms and lead future thinking.

\section{Conclusions}

Scientometric results show that the publication record for water-related research in South Africa contained 6007 publications from 1977 to 2011. WRC research reports amounted to $1760(29.30 \%)$ of this total. The remainder were peer-reviewed journal articles. Of these journal articles, $1829(30.45 \%)$ were published in Water SA. The publication record also increased in number dramatically since 1990, with more articles being published annually than each previous year throughout the data set.

Paradigms were identified through the scientometric mapping methods using the publication record to show a history of water research from 1977 to 2011. Overall, the research output focused predominantly on management, development, models, quality and system treatment. Technical matters are dominant in the historical record but other paradigms such as allocative efficiency, uncertainty and risk are also present to lesser degrees. The change in paradigms is observed when these results are examined over successive time periods.

Two major paradigm approaches were observed in the analysis of water research publications along with one significant transition period. The first set of paradigms, from 1977 to 1991, emphasises the hydraulic mission in which research and implementation aimed to secure supply and understand basic natural systems. This period is dominated by engineering and laboratory-related disciplines. The 'getting more' and 'supply management' paradigms are characterised by efforts to ensure water supply, drainage and the development of the sewered city - mainly engineering and biological-related research efforts. In the following 10 years (1992-2001), there is a transition in which quality constraints and fields of management and planning become prominent. This paradigm is in response to changes in water deficits and a focus on end-use efficiency. A second transition occurs with a new social contract around water at a time when the new political regime enters government in a period of democratic transition, growing environmentalism and a rise of civil society activism. The need to plan, model catchments and include other disciplines becomes evident in the research environment.

The question prioritisation activities using horizon scanning methods provided an opportunity to engage with a wide and diverse population of water research stakeholders and practitioners. The survey resulted in a substantial collection of research questions from water stakeholders and researchers. Many questions deal with immediate to mediumterm concerns while only a few aim to tackle long-term or systemic problems. Others are coupled or integrated questions that cover a number of disciplines.

There are recognised limitations to this study. The simplification of scientometrics causes a potential loss in detail and context. The interpretations of output maps remain subjective but the method does provide powerful, macro perspectives of a research area. However, albeit simplistic, the methods used in the field of scientometrics are repeatable and are not dependent on the choice of experts and their opinions which may vary as the choice of the participants changes in peer reviews. ${ }^{33}$

It is recommended that further detailed mapping and analysis be done on publications to explore the reasons that might cause paradigm shifts as well as to understand what is missing in the existing body of knowledge. Horizon scanning has many inappropriate elements for the South African context as it is limited to a degree by its reach and participation. It is recommended that further prioritisation activities are undertaken to guide research but that these are expert lead and informed at the earliest stage before taking the results to a wider audience for consultation. The current state of questioning does, however, provide an overall perspective of what a large and diverse group of research stakeholders and practitioners believes is important, even if these may not deal with long-term challenges but are rather more situated in addressing current and pressing research needs.

\section{Acknowledgements}

This publication was based on the Water Research Commission funded project $\mathrm{K} 5 / 2170$. The project leaders wish to thank the reference group participants, the workshop delegates who gave of their time to prioritise research questions and debate research in South Africa and the project staff who contributed in their specific tasks and fields.

\section{Authors' contributions}

R.S. performed most of the experiments and wrote the manuscript; K.W. was the project leader; and I.J. was the funding manager and expert advisor.

\section{References}

1. Tempelhoff J, Hoag H, Ertsen M. Water history and the modern. Water History. 2009;1:81-82. http://dx.doi.org/10.1007/s12685-009-0014-3

2. Tewari D. A detailed analysis of evolution of water rights in South Africa: An account of three and a half centuries from 1652 AD to present. Water SA. 2009;35:693-710. http://dx.doi.org/10.4314/wsa.v35i5.49196

3. Allan T. Water in international systems: A risk society analysis of regiona problemsheds and global hydrologies. Presented at: The 1999 Oxford University Conference on Water Resources and Risk; 1999 March 22; Oxford, UK. Available from: http://awiru.co.za/pdf/allantony2.pdf

4. Republic of South Africa (1971) Water Research Act No. 34 of 1971. Pretoria: Government Printers.

5. Republic of South Africa (1998) National Water Act No. 36 of 1998. Pretoria: Government Printers.

6. Herold C. The water crisis in South Africa. 2009 Des Midgley Memoria Lecture presented at the 14th SANCIAHS Symposium; 2009; University of KwaZulu-Natal, Pietermaritzburg, South Africa.

7. Funke N, Nortje K, Findlater K, Burns M, Turton A, Weaver A, et al. Redressing inequality: South Africa's new water policy. Environment. 2007;49(3):10-23. http://dx.doi.org/10.3200/ENVT.49.3.10-25 
8. Schreiner B. Water services: Yesterday, today and tomorrow - a strategic perspective. In: Proceedings of the 2006 Water Institute of South Africa Biennial Conference; 2006 April 21-25; Durban, South Africa. Johannesburg: WISA; 2006. Available from: http://www.ewisa.co.za/literature/files/331\%20 Schreiner.pdf

9. Fallenmark M, Rockstrom J. The new blue and green water paradigm: Breaking new ground for water resources planning and management. J Water Res PI-ASEC. 2006;132(3):129-132. http://dx.doi.org/10.1061/(ASCE)07339496(2006)132:3(129)

10. Siebrits R, Winter K, Barnes J, Dent M, Ekama G, Ginster M, et al. Priority water research questions for South Africa developed through participatory processes. Water SA. 2014;40(2):199-209. http://dx.doi.org/10.4314/wsa. v40i2.2

11. Kuhn T. The structure of scientific revolutions. Chicago, IL: University of Chicago Press; 1962.

12. Turton A. The role of science in deepening democracy: The case for water in post-apartheid South Africa. J Transdiscipl Res S Afr. 2009;5(1):9-28.

13. Van Vuuren L. What's in a name: Looking back at the start of public water governance. The Water Wheel. 2009;July/August:38-41.

14. Ohlsson L, Turton A. The turning of a screw: Social resource scarcity as a bottle-neck in adaptation to water scarcity. Stockh Water Front. 2000;1:10-11.

15. Allan J. Water in the environment/socio-economic development discourse: Sustainability, changing management paradigms and policy responses in a global system. Gov Oppos. 2005;40(2):181-199. http://dx.doi.org/10.1111/ j.1477-7053.2005.00149.x

16. Hood W, Wilson C. The literature of bibliometrics, scientometrics and informetrics. Scientometrics. 2001;52(2):291-314. http://dx.doi. org/10.1023/A:1017919924342

17. Tague-Sutcliffe J. An introduction to informetrics. J Inf Process Manage. 1992;28:1-3. http://dx.doi.org/10.1016/0306-4573(92)90087-G

18. Klavans R, Boyack K. Quantitative evaluation of large maps of science. Scientometrics. 2006;6(3):475-499. http://dx.doi.org/10.1007/s11192006-0125-x

19. Rafols I, Leydesdorff L. Content-based and algorithmic classifications of journals: Perspectives on the dynamics of scientific communication and indexer effects. J Assn Inf Sci Technol. 2009;60(9):1823-1835. http://dx.doi. org/10.1002/asi.21086

20. Roessner D. Quantitative and qualitative methods and measures in the evaluation of research. Res Evaluation. 2000;9(2):125-132. http://dx.doi. org/10.3152/147154400781777296
21. Rafols I, Porter A, Leydesdorff L. Science overlay maps: A new tool for research policy and library management. J Assn Inf Sci Technol. 2010;61(9):1871-1887. http://dx.doi.org/10.1002/asi.21368

22. Sutherland $W$, Woodroof $H$. The need for environmental horizon scanning. Trends Ecol Evol. 2009;24(10):523-527. http://dx.doi.org/10.1016/j. tree.2009.04.008

23. Shackleton C, Scholes B, Vogel C, Wynberg R, Abrahamse T, Shackleton S, et al. The next decade of environmental science in South Africa: A horizon scan. S Afr Geogr J. 2011;93(1):1-14. http://dx.doi.org/10.1080/0373624 5.2011 .563064

24. LaRowe G, Ambre S, Burgoon J, Ke W, Borner K. The scholarly database and its utility for scientometrics research. Scientometrics. 2009;79(2):219-234. http://dx.doi.org/10.1007/s11192-009-0414-2

25. Todrov R. Representing a scientific field: A bibliometric approach. Scientometrics. 1989;15(5):593-605. http://dx.doi.org/10.1007/BF02017072

26. Small $H$. Macro-level changes in the structure of co-citation clusters Scientometrics. 1993;26(1):5-20. http://dx.doi.org/10.1007/BF02016789

27. Janssens F, Leta J, Glanzel W, De Moor B. Towards mapping library and information science. Inf Process Manage. 2006;42:1614-1642. http://dx.doi. org/10.1016/j.ipm.2006.03.025

28. Wallin J. Bibliometric methods: Pitfalls and possibilities. Basic Clin Pharmacol Toxicol. 2005;97:261-275. http://dx.doi.org/10.1111/j.1742-7843.2005. pto_139.x

29. Borner K, Mane K. Mapping topics and topic bursts in PNAS. Proc Natl Acad Sci. 2004;101(1):5287-5290. http://dx.doi.org/10.1073/pnas.0307626100

30. Noyons E, Van Raan E. Monitoring scientific developments from a dynamic perspective: Self-organized structuring to map neural network research. J Assn Inf Sci Technol. 1998;49(1):68-81.

31. Turton A, Meissner R. The hydrosocial contract and its manifestation in society: A South African case study. In: Turton A, Henwood R, editors. Hydropolitics in the developing world: A southern African perspective. Pretoria: African Water Issues Research Unit; 2002.

32. Sutherland W, Fleishman E, Masica M, Pretty J, Rudd M. Methods for collaboratively identifying research priorities and emerging issues in science and policy. Methods Ecol Evol. 2011;2(3):238-247. http://dx.doi. org/10.1111/j.2041-210X.2010.00083.x

33. Pouris A. Peer review in scientifically small countries. R\&D Manage. 1998;18(4):333-340. http://dx.doi.org/10.1111/j.1467-9310.1988.tb00608.x 